\title{
Metodología y práctica en programas de inmersión
}

\author{
Josu Sierra
}

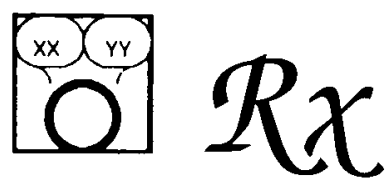

Los exitosos programas de inmersión ban resultado un gran descubrimiento en el mundo del aprendizaje de lenguas en muchos países, y especialmente en aquellos en que existen lenguas minorizadas. En estos casos, muchas veces se está viviendo una «época dorada» en el desarrollo del bilingüismo y la normalización lingiüstica impulsadas por este nuevo enfoque a la bora de aprender una segunda o tercera lengua.

\section{INTRODUCCION}

Los programas de inmersión, nacidos como reacción a los programas-asignatura en el ámbito escolar, aportan una ingente cantidad de investigación, de tipo cuantitativo, sobre todo (Rebuffot, 1993:82). La necesidad de centrarse, en adelante, en las variables del proceso de aprendizaje ha sido puesta de relieve más de una vez por varios autores (Arnau, 1993:89).

No obstante, y mientras esto se lleva a cabo, es imprescindible impulsar la reflexión acerca del trabajo cotidiano de los profesionales en los programas de inmersión, ya que, a veces, no sabemos qué es exactamente lo que hacemos y porqué lo estamos haciendo así.

De momento, puede ser interesante recopilar y analizar los principios metodológicos de los programas de inmersión, tanto los claramente enunciados como los no suficientemente explicitados, así como las consecuencias de la metodología aplicada a lo largo de tantos y tan fructíferos años.

Citemos en primer lugar los principios teóricos de los programas de inmersión de la mano de Safty (Safty, 1991:473), para, posteriormente, comentar las incidencias de la metodología aplicada según los estudios disponibles. Tras analizar los problemas derivados de la metodología de inmersión incidiremos en las posibles soluciones y también consideraremos otros factores al margen de los puramente metodológicos, tales como los de la estructura de la clase e, incluso, el profesor/a.

\section{PRINCIPIOS TEÓRICOS}

1, Exposición temprana e intensiva a la L2; 2 , Construcción del «Entorno Imitativo Natural» en el proceso de adquisición-aprendizaje de la L2; 3, Interferencia y Transferencia entre las lenguas; 4, Enseñanza Contextualizada, y 5, Estrategia Comunicativa. 


\section{Exposición temprana e intensiva a la L2}

Normalmente, los programas de inmersión garantizan una exposición temprana, en el caso de inmersión temprana, e intensiva a la 2. lengua.

No obstante, también los programas de inmersión tardía han demostrado su eficacia y en numerosos estudios los alumnos «mayores», en un contexto escolar, han logrado niveles determinados más rápidamente que los «jóvenes».

También es cierto, y es comúnmente aceptado, que es necesario el aprendizaje precoz para llegar a dominar con menor esfuerzo algunos aspectos de la lengua. Aspectos más bien informales, tales como pronunciación, entonación, acento, etc. Pero en aspectos más formales, es crucial la importancia del desarrollo cognitivo, por lo que determinadas destrezas se adquieren más fácilmente en edades más avanzadas.

La ventaja de la precocidad esta, por tanto, condicionada por otros aspectos como la utilización de metodologías centradas en la comunicación y en los aspectos informales de la lengua y, por supuesto, por las características y motivación de los sujetos.

Baker dice, citando a Singleton, (Baker, 1993:75) que en una situación formal como la del aula, los alumnos mayores, al principio, aprenden más rápido que los jóvenes. Sin embargo, la duración de la exposición (años aprendiendo) también es una factor importante en el aprendizaje de la lengua. Por tanto, los niños que comenzaron en la Educación Infantil, consiguen mejores niveles de competencia que quienes han empezado más tarde, a pesar de que los «alumnos tardíos» pueden alcanzar también muy buenos niveles, sobre todo si están muy motivados.

Al fin y al cabo, no existe un "periodo crítico» para la implantación del aprendizaje de la $2^{\mathrm{a}}$ lengua en la escuela ya que el proceso de adquisición-aprendizaje suele ser el fruto de la interacción de múltiples variables.

Además de una exposición precoz a la segunda lengua, también la exposición intensiva es propia de los programas de inmersión.

Es necesario garantizar una mínima cantidad de input y una progresión tanto en la cantidad como en la calidad. De lo contrario, los alumnos/as tendrán grandes dificultades en avanzar hasta niveles adecuados y se verán condenados a empezar de cero curso tras curso.

El nivel mínimo de input no se consigue con 2 o 3 horas semanales de clase. Aun utilizando una metodología adecuada, es preciso asegurar una presencia de la lengua y una progresión de esta presencia para garantizar unos resultados mínimos. He ahí el ejemplo de algunos modelos B (inmersión parcial) en el País Vasco: no se consigue llegar a un nivel de L2 suficiente y esto puede provocar problemas en el rendimiento académico. También en las clases de lengua extranjera ocurre que no es suficiente con utilizar modernás metodologías ni con aprender la lengua durante varios años para alcanzar un nivel «umbral» que permita desarrollar la L3 si no se llega a un nivel suficiente de input y a un aumento progresivo de la presencia de la lengua en cuestión.

Del mimso modo, en modelos de inmersión parcial canadienses también ha aparecido este problema (Hamers, Blanc, 1983:319) al comprobar el escaso nivel de L2 que consiguen los sujetos en comparación con los pertenecientes a programas de inmersión total.

\section{Construcción de un entorno imitativo natural para la adquisición-aprendizaje de la L2}

En los programas de inmersión se trata de que la $2^{\mathrm{a}}$ lengua se aprenda de modo similar a como se aprende la primera, por lo que se intenta construir un Entor- 
no Imitativo Natural, cuyas características, en el caso de la $1^{\text {a }}$ Lengua, son las siguientes:

a) Exposición permanente a la lengua.

b) Los padres, modelos a imitar, utilizan un lenguaje adaptado para comunicarse con el niño/a y, progresivamente, van introduciendo frases más complejas a medida que el niño/a va adquiriendo un mayor nivel de comprensión oral.

c) La comprensión lingüística es previa a la producción.

d) Cultura y lengua van inseparablemente unidos, reforzándose mutuamente.

e) El nivel socioeducativo y sociocultural de los padres influye en el entorno lingüístico del niño/a y marca el proceso de adquisición-aprendizaje de la $1^{\mathrm{a}}$ lengua.

f) El aprendizaje de la $1^{a}$ lengua en casa es un proceso de ensayo-error.

Los programas de inmersión, por supuesto, no pueden reproducir exactamente este entorno y, además, en un contexto formal como la escuela, no pueden darse una serie de condiciones antes descritas. No obstante, en la medida de lo posible, plantean la adquisición-aprendizaje de la $2^{\mathrm{a}}$ lengua en términos parecidos, es decir, aprender la lengua en una comunicación real producida en situaciones comunicativas reales.

\section{Interferencia y transferencia entre las lenguas}

La transferencia e interferencia entre las lenguas son mecanismos básicos en los programas de inmersión.

El niño/a parte de la $1^{\text {a }}$ lengua, prestigiosa y socialmente mayoritaria en la mayoría de los casos, y al contactar con la $2^{2}$, ocurre un proceso de doble dirección:

- Interferencia: La estructura de la lengua de la que se parte, la sintaxis, el léxico, etc., pueden llegar a contaminar la otra e impedir su adquisición-aprendizaje a causa de la hipergeneralización de normas, estructuras, fonética..., aunque ello mismo tiene su parte positiva en la

- Transferencia: Es un proceso muy general, en el que el sujeto ya conoce una lengua y parte de los esquemas de esta misma lengua, muchos de ellos comunes a su vez a muchas otras lenguas e incluso a todas las lenguas, para aprender la $2^{\mathbf{a}}$. Los elementos que las dos lenguas en cuestión poseen en común son automáticamente compartidos, impulsando la adquisición de la L2.

Las similitudes o diferencias entre lenguas marcan de alguna manera este proceso, aunque hay otros muchos factores intervinientes que relativizan las semejanzas como el estatus de las lenguas o su prestigio y la motivación de los aprendices, por ejemplo.

En palabras de Wong Fillmore (Wong Fillmore, 1991:56):

"Este tipo de conocimiento y experiencia lingüistica previa puede ayudar a los aprendices de la $2^{2}$ lengua a descubrir los medios para realizar las mismas funciones en la nueva lengua. En otras palabras, ellos están guiados en sus esfuerzos de aprendizaje de la lengua por lo que saben que es posible y útil a partir de su conocimiento de la $1^{a}$ lengua. Por tanto, el aprendiz de la $2^{a}$ lengua parte con una idea bastante buena de qué buscar en el nuevo idioma.

El hecho de que los alumnos presuponen que las formas que se encontrarán en la L2 pueden ser funcionalmente equivalentes a las de la L1, puede llevar a los aprendices a adquiritlas más eficientemente que si fuese de otro modo. Al mismo tiempo, sin embargo, este hecho puede interferir también con el aprendizaje, ya que esta presuposición lleva a veces a los alumnos a extraer consecuencias, en gran medida injustificadas, de que las formas de la L2 son estructural y funcionalmente idénticas a las 
formas y usos de la L1. Cuando esto ocurre, nos encontramos con interferencia o transferencia de la $1^{a}$ lengua. En resumidas cuentas, no obstante, el resultado de este proceso es positivo."

Para ilustrar la importancia del proceso de transferencia, citaremos un caso de inmersión parcial en Estados Unidos:

En una experiencia llevada a cabo en Fairfax, Virginia, se estudiaron los resultados de un programa de inmersión parcial en japonés. A pesar de estudiar las matemáticas en japonés en el nivel 1 , los sujetos de este programa consiguen mejores resultados en las pruebas de matemáticas que los alumnos del programa normal. Este resultado se ha probado por primera vez con una lengua cuyo alfabeto no es romano(Thomas, Collier, Abbott, 1993:170-179).

\section{Aprendizaje contextualizado de la L2.}

El aprendizaje contextualizado es, seguramente, la característica más definitoria de los programas de inmersión, que siguen una estrategia EXPERIENCIAL frente a otras estrategias ANALÍTICAS (Harley, 1993:246), sin que ello signifique la total ausencia de INSTRUCCIÓN DE LA L2 en muchos programas de inmersión.

En el enfoque metodológico de la inmersión, el proceso de aprendizaje no se centra en la L2 sino en el aprendizaje del CONTENIDO académico por medio de la $2^{2}$ lengua. La lengua es un instrumento y el objetivo es la utilización real de la misma a la hora de aprender contenidos. Esto da significado a la lengua, no se habla sobre la lengua sino que se aprende utilizándola.

En palabras de Vila (Vila, 1993:163), «En esta perspectiva, de lo que se trata es de enseñar la lengua en relación a los usos que se requieren en el contexto social e institucional de enseñanza-aprendizaje, de modo que aprendiendo a usar el lenguaje se posibilita, a su vez, su aprendizaje como instrumento de la inteligencia representativa....... Desde la perspectiva instrumental de la enseñanza de la lengua, el énfasis, como ya hemos dicho, se situa en relación a la realización conjunta alumno-profesor de tareas que requieren uso del benguaje, de modo que el alumno tenga algo que decir y el profesor esté interesado en lo que dicen».

\section{Estrategia comunicativa}

La estrategia comunicativa es una característica absolutamente unida a la anterior. La segunda lengua se aprende utilizándola en verdaderas situaciones comunicativas. El aprendiz debe sentir la necesidad de utilizar la $2^{2}$ lengua en situaciones comunicativas reales (Ellis, 1985:161) y ello es posible, al menos teóricamente, en los programas de inmersión gracias a la metodología centrada en el contenido, lo cual dota de sentido y significado real a la lengua y posibilita situaciones comunicativas en las que puede sentirse la necesidad de comunicarse en la L2.

Artigal dice que «En definitiva, la adquisición de una nueva lengua no puede ser entendida como una simple exposición al «input», sino como un proceso de.reconocimiento/construcción de este «input», como un proceso mediante el cual el aprendiz debe llenar progresivamente de significado las formas que recibe en la lengua objeto de aprendizaje. A la vez, este progresivo proceso de construcción semiótica debe incluir «momentos sociales», es decir, momentos en los que dicho proceso dependa de la interacción con un «otro» capaz de organizar territorios semióticos que resulten reconocibles y, en consecuencia, compartibles por todos los participantes». (Artigal, 1993:111). 


\section{ALGUNOS PROBLEMAS EN LA PRÁCTICA}

Hasta aquí la exposición de los principios teóricos subyacentes a los programas de inmersión, pero, como el resto de los programas, éstos no son capaces de solucionar todos los problemas en el aprendizaje-adquisición de lenguas. En la practica diaria han aparecido varias disfunciones y efectos inesperados de los que hablaremos a continuación.

\section{El tiempo de exposición}

El tiempo de exposición a la $2^{a}$ Lengua no es un valor absoluto. El tiempo necesita de la concurrencia de otros factores para que resulte efectivo (Sierra, 1993:30). Más importante que el «Cuanto» es el «Cómo» se hacen las cosas. La clave son las características del input recibido. En un determinado momento, el aumentar el tiempo de exposición a la L2 no tendría efecto si no se cumpliesen otro tipo de condiciones, lo que nos lleva a analizar las características del input.

\section{Input: cantidad y calidad}

Krashen dijo que no era suficiente la mera exposición a la lengua para aprender una $2^{a}$ lengua. El aprendiz necesita un «input comprensible» para llegar a la «Adquisición de la Segunda Lengua». Este input, para ser comprensible, debe ser algo más de lo que el sujeto es capaz de entender, «i +1», (Krashen, 1982:21). La adquisición tiene lugar cuando el aprendiz comprende la lengua que contiene «i+1». No obstante, el alumno no asimilara más que un porcentaje de ese input, denominado «intake».

Todo esto supone que será necesaria una gran cantidad de input y que este debe ser progresivamente más complejo para llegar a la adquisición de la L2. No se trata de exponer a sujeto a un nivel que pueda entender o a otro que no pueda comprender sino de secuenciar la complejidad del input que el aprendiz recibe, de modo que sea progresivo.

Sin embargo, a pesar de cumplirse estos requisitos, la práctica diaria de los programas de inmersión nos dice que siguen existiendo problemas en la adquisición de la $2^{\mathrm{a}}$ lengua, algunos de ellos, derivados de la propia metodología de inmersión, como veremos.

\section{El output, complemento imprescindible del input}

Si los programas de inmersión se centran en el CONTENIDO y no en la $2^{\mathrm{a}}$ lengua, es decir, en el significado y no en la forma, los efectos de este planteamiento son visibles en varios aspectos, tal y como Swain nos demuestra en sus estudios.

Para conseguir la adquisición-aprendizaje de una $2^{\circ}$ lengua es imprescindible el input comprensible, pero NO ES SUFICIENTE. El OUTPUT comprensible es también necesario para cumplir este objetivo. Del simple análisis semántico, el aprendiz debe llegar al análisis sintáctico de la lengua (Swain, 1985:252).

Según Merril Swain, existen una serie de «lagunas» en los programas «CENTRADOS EN EL CONTENIDO» o en la «actividad» (Swain, 1988:68-83):

1. La enseñanza está, normalmente, totalmente orientada hacia la comprensión del significado. Ello puede producir el fenómeno denominado «Selective Listening", es decir, es posible captar el mensaje, entenderlo, pero sin hacer un análisis sintáctico y morfológico. ¿Como? Pues, por ejemplo, gracias al léxico combinado 
con información extralinguística. Sin siquiera conocer la sintaxis y la morfología de la lengua, puede llegar a comprenderse el significado con la ayuda de algunas palabras y comunicación no-verbal.

2. Centrando totalmente la enseñanza en el «Significado», el profesor o la profesora da a los alumnos, frecuentemente, mucha información inconsistente y aleatoria. La corrección de los errores de los alumnos, por ejemplo, se lleva, al parecer, de modo aleatorio y arbitrario en muchos programas de inmersión.

Según estudios realizados al respecto, en algunos programas solamente se corregía el $19 \%$ de los errores de los alumnos y, muchas veces, esta corrección se hacía asistemáticamente y de modo bastante desorganizado (Allen, Swain, Harley, Cummins, 1990:67). Como ejemplo, en algunas ocasiones, la corrección de los errores dependía del «nivel de enfado del profesor».

Cada profesor tiene sus prioridades y sus propios modos de actuación. Algunos creen que su cometido es enseñar contenidos y que los errores del lenguaje deben corregirse en la clase de lengua y, por tanto, solamente corrigen los errores referentes al contenido. Otros, en cambio, no tienen más remedio que corregir los errores de lenguaje, ya que estos ponen en riesgo la adquisición de contenidos (Chaudron, 1986:75).

3. El input que el alumno/a recibe puede ser «Funcionalmente restringido», es decir, que ciertas formas y usos de la lengua, quizá no aparezcan nunca en el discurso del aula. Algunas estructuras, giros, verbos, léxico, absolutamente imprescindibles en situaciones naturales puede que jamás aparezcan en el aula, o lo hagan en muy pocas ocasiones, limitando la capacidad comunicativa de los aprendices y dificultando seriamente el uso de la L2 fuera del contexto escolar.

Muchas veces, la propia opinión del profesor acerca de la lengua que utiliza normalmente en clase suele estar bastante apartada de la realidad, no siendo consciente de este problema de la limitación funcional del input.

4. Según los estudios de algunos investigadores, en muchos modelos de «enseñanza de la $2^{\mathrm{a}}$ lengua centrada en el contenido», prácticamente el único que habla en clase es el profesor (Allen, Swain, Harley, Cummins, 1990:66). En las aulas, el mecanismo por antonomasia es el de pregunta-respuesta y el $40 \%$ de la producción de los alumnos en algunos programas de inmersión se limita a una sola palabra.

\section{BUSCANDO SOLUCIONES}

1. La solución contra el «Selective Listening», en opinión de Swain (Swain, 1988) es el impulsar LA PRODUCCIÓN. Muchas veces entendemos el discurso sin un exacto conocimiento sintáctico y morfológico pero es muy difícil producir en la L2 sin ese nivel de conocimiento. El alumno, además de en el significado, debe centrarse en la forma también y el empujarle a producir en la L2 es una eficaz medida para conseguirlo.

Ellis, no obstante, relativiza el efecto que la participación del alumno o la producciọ́n puedan tener en la adquisición de la L2 y matiza que la relación entre participación y adquisición depende de muchos factores a tener en cuenta como la personalidad del aprendiz, el nivel de competencia en la L2 que ha alcanzado, si la producción es voluntaria u obligada, la longitud de la producción y la necesidad del alumnos para expresarse precisa y coherentemente (Ellis, 1992:46).

2. La solución en la corrección de errores es también bastante matizada. El problema no se soluciona practicando la sistematicidad y cortando la comunicación sino integrando fórmulas de corrección en el discurso comunicativo, tales como repeticiones, matizaciones o contextualizaciones integradas en el hilo de la comuni- 
cación. Tiene un papel importante en la corrección de errores, fosilizaciones, generalizaciones indebidas...etc. de los alumnos la utilización también de una estrategia analítica en estrategias experienciales, programas centrados en el contenido o en la actividad, como complemento indispensable para asegurar la adquisición de la L2 en el contexto escolar (Harley, 1993:254). Forma parte de esta estrategia el hacer conscientes a los alumnos de las diferencias entre la L1 y la $\mathrm{L} 2$ en determinado tipo de estructuras cuyo uso es incorrecto, el promover el análisis de las diferencias entre una lengua y otra en este caso y en características infrecuentes, irregulares o dificultosas para hablantes de la L1.

La estrategia analítica es apropiada, según Harley, en cualquier estadio de un programa de aprendizaje en la L2, cuidando no sobreestimar las capacidades lingüísticas y metalingüísticas de los alumnos y subordinada siempre a la naturaleza de la tarea que se esta desarrollando.

3. Respecto al input funcionalmente restringido, habría que analizar primeramente cual es la lengua que utiliza el profesor o la profesora. Una buena medida puede ser el grabarse su propio discurso y analizarlo, por ejemplo. De este modo se han descubierto muchos problemas: la no utilización del tiempo pasado en clases de Sociales, la nula aparición de verbos de acción en la interacción del aula, la omnipresencia del imperativo en muchas actividades, la no adecuación del lenguaje a ciertas situaciones sociales por el hecho de que nunca se han visto en clase, etc.

Las soluciones, no obstante, no vendrán solamente de la autoconciencia del profesor, también son importantes los recursos didácticos adoptados para el tratamiento de los temas, Ellis atribuye una gran importancia al control de los temas por parte del alumno, en grupo pequeño, por ejemplo (Ellis, 1992:44), pero, sin duda, es tremendamente efectiva la interacción con sujetos nativos, o incluso con alumnos más adelantados, con un nivel superior, tal y como puede verse en algunos estudios (Porter, 1986:219). Es importante que los alumnos interaccionen con alguien que posee un nivel de L2 superior al suyo (mejor si, ademas de un nivel superior, posee otras cualidades como capacidad de adaptación al nivel del interlocutor...etc.) para un desarrollo efectivo del proceso de adquisición-aprendizaje. En definitiva, serían iniciativas eficaces las que potenciasen la necesidad comunicativa que se da en las situaciones naturales y las que proporcionan al aprendiz la posibilidad de interaccionar libremente en la $2^{a}$ lengua (Ellis, 1985:161).

4. Para impulsar la producción en el aula, por fin, las soluciones vendrán de la sustitución del sistema de pregunta-respuesta existente. El trabajar en programas centrados en el contenido, muchas veces, limita en gran medida, sobre todo a partir de ciertos niveles, el proceso de adquisición-aprendizaje de la $2^{\mathrm{a}}$ lengua. Se considera, a veces, que los alumnos ya son «hablantes nativos» y se actúa como si lo fuesen: «Ya han aprendido la $2^{\mathrm{a}}$ lengua. De aquí en adelante nos centraremos en el contenido». Este punto de vista es absolutamente irreal.

Recordaremos las palabras de Swain para recapitular lo que hemos comentado (Swain, 1988:81):

"Resumiendo, be intentado mostrar que la típica enseñanza del contenido no es necesariamente una buena enseñanza de la segunda lengua. Generalmente, la enseñanza de contenidos se centra en la comprensión del significado. Sin embargo, lo que el aprendiz de la segunda lengua necesita es centrarse sobre la relación forma-significado. Ello se vería facilitado a través de la producción del lenguaje, ya sea en forma oral o escrita. Teniendo en cuenta que la típica secuencia preguntalrespuesta presente en las clases de contenido tiende a producir respuestas cortas de minima complejidad por parte de los alumnos, al menos parte del contenido de la clase debe ser sustituido por actividades que exijan un lenguaje más largo, más complejo y coherente por parte de los aprendices". 
Las actividades que cita pueden plantearse de muchas maneras. Trabajo en grupos, intercambio de correspondencia, programas de radio o TV escolares, publicaciones de la clase, representaciones, entrevistas, juegos, confección de artículos, murales o conferencias de los alumnos...etc.

\section{FACTORES COMPLEMENTARIOS A LA METODOLOGÍA}

La aplicación de cualquier tipo de metodología, que quiera obtener buenos resultados, exige llevar a cabo un proceso de adaptación a la situación concreta de cada cual. La composición del aula, tanto por la procedencia de los alumnos y sus características, los diferentes niveles de L2 que poseen, las diferencias socioeconómicas, etc. y las características del profesor o profesora: personalidad, nivel de dominio de la lengua, capacitación profesional, recursos...., son variables fundamentales a la hora de organizar la clase.

Seguiremos en este sentido las investigaciones de Wong Fillmore por su indudable interés practico (Wong Fillmore, 1982:283-296, 1985:17-50)

\section{Organización de la clase según sus características}

Simplificando, hay, generalmente, dos tipos de organización entre las que se mueven todas las posibles organizaciones: «la centrada en el profesor» y «la centrada en el alumno". En la primera, la mayor parte de las actividades están organizadas para el gran grupo y el profesor es quien las dirige. En la segunda, son mayoría las actividades individuales y de pequeño grupo, los alumnos trabajan en común y la implicación del profesor es menor.

Las clases del segundo tipo o más abiertas se adaptan mejor a situaciones en las que existe una mezcla de alumnos nativos con aprendices para los cuales esta lengua es su $2^{a}$ lengua, o a alumnos no principiantes. La aplicación de un esquema excesivamente abierto entre alumnos aprendices de $2^{\text {a }}$ lengua puede originar, a través de la interacción libre entre ellos, la aparición de «gramáticas internas» etc.

Por otro lado, las clases dirigidas por el profesor o la profesora, son más controladas pero adolecen de los problemas anteriormente vistos si es que no se valen de otras organizaciones más abiertas que posibiliten la producción y la interacción. Lo más práctico, por tanto es, dependiendo de la composición del alumnado y de las capacidades del profesor, mantener un equilibrio organizativo que posibilite los diversos planteamientos en función de la actividad y del objetivo perseguido.

\section{Características estructurales de las clases}

a) Clases formales con delimitaciones claras:

Según algunos estudios, las clases más exitosas suelen ser muy consistentes en lo que se refiere a la organización, es decir, el alumno o la alumna sabe lo que se va a hacer y conoce los pasos que se dan para hacerlo, existe una rutina diaria bien definida y unas pautas muy delimitadas.

b) El formato de la clase tiene fases claras y cada fase su propio lenguaje:

El profesor presenta cada fase de la clase de modo diferenciado y cada fase tiene su propia rutina. De esta manera, las actividades resultan familiares a los alumnos y colaboran en la creación de un contexto conocido que facilita tanto la comprensión como la participación y la producción.

c) Clases con cauces y mecanismos de participación: 
Otra característica de las clases exitosas es la implantación de turnos para la participación de los alumnos y alumnas. Esto afecta a la interacción en la lenguaobjetivo, a la motivación del alumno, al comportamiento del grupo etc.

En una fase determinada pueden pedirse voluntarios para determinada actividad, en otra puede procederse sistemáticamente, por orden o aleatoriamente, a preguntar o encargar una tarea. El profesor debe utilizar variados mecanismos que impulsen la participación pero siguiendo un procedimiento claro en cada fase.

\section{Qué lengua utilizar en clase}

Sabemos que la "comprensividad" del input es decisiva a la hora de producir la adquisición-aprendizaje de la L2. La lengua esta funcionando como input cuando - posibilita una verdadera función comunicativa y no cuando el alumno está comprendiendo el significado por el contexto. La negociación del significado es el mecanismo que posibilita la adquisición-aprendizaje.

¿Cómo hacer entonces que la lengua del profesor funcione como input?

a) Con una clara separación de las lenguas en caso de que se deban utilizar la L1 y la L2, por medio de diferentes espacios, diferentes tiempos, diferentes profesores.

b) Subrayando la comunicación y la comprensión, adaptando el contenido y la presentación del mismo a la situación de los aprendices.

c) Cuidando la gramaticalidad y adecuación de la lengua utilizada en clase y evitando el «habla extranjera» o la inadecuación del lenguaje en aras de la comprensión por parte de los alumnos y alumnas.

d) La utilización de esquemas y rutinas en el sentido más arriba citado.

e) La utilización de repeticiones dando opción a los aprendices a escuchar las mismas o parecidas estructuras más de una vez.

f) La adaptación de la participación del alumno a las características y necesidades de cada uno.

g) La riqueza del lenguaje utilizado, ya que la comprensibilidad no debe suponer artificialidad o «lenguaje ortopédico», sino riqueza y claridad a la vez.

\section{LA VARIABLE PROFESOR}

A la hora de explicar los resultados de cualquier programa de aprendizajeadquisición de una lengua en el ámbito escolar, es primordial la consideración de la variable profesor/a.

Cuando decimos PROFESOR o PROFESORA nos estamos refiriendo, en realidad, a múltiples aspectos que abarcan desde su dominio de la lengua-objetivo hasta sus pautas de comportamiento en el grupo, e incluso su implicación afectiva, pasando por su capacitación profesional y sus recursos didácticos.

Esto es, al menos, lo que se desprende de un estudio de Leeman Guthrie (Leeman Guthrie, 1987) aplicable también a programas de inmersión.

Observando seis profesores/as diferentes en clase de francés como lengua extranjera, se tuvieron en cuenta diversos aspectos de su discurso en clase, como la utilización de la L1 o la L2 (en qué porcentaje), la producción oral del alumno, las actividades centradas en la forma o en un contenido, el esquema de las clases, las oportunidades y modalidades de participación del alumno/a, las repeticiones, correcciones y el entorno afectivo creadọ por el profesor/a entre otros. 
Como conclusión, la autora dice que «Lo que parece claro en esta discusión es que el entorno óptimo de la clase para el aprendizaje de la lengua no puede ser caracterizado en términos de simple generalización de temas, técnicas de aprendizaje, tipos de actividades o incluso distribución del lenguaje.... /... Parece probable que la adecuación de la clase como entono de adquisición del lenguaje, dependa de una compleja trama de factores lingüísticos, afectivos y mecánicos interdependientes y no todos ellos significativos fuera del discurso de la clase. A la hora de discutir acerca de la clase como entorno de adquisición del lenguaje, hemos de tener en cuenta circunstancias únicas de la misma - la naturaleza estructurada del discurso del aula, la limitada opción de interlocutores, el efecto del soporte escrito sobre la interacción hablada etc. Y debemos reconocer que algunas condiciones básicas del discurso natural, como el mutuo acuerdo de los participantes de entrar en la conversación, no pueden darse por hechas en el aula. A través de la observación de la interacción en la clase... ... podemos quizá reconocer una serie de factores intervinientes, independientes de cualquier metodología estrictamente definida, que contribuyen a crear un óptimo entorno en la clase.»

En definitiva, no es posible predecir el resultado de la actuación de un profesor/a en la clase basándose únicamente en aspectos parciales como su nivel de L 2 o el dominio de cierta metodología. El resultado será explicado por la combinación de múltiples factores, citados más arriba, combinación que permite la obtención de buenos resultados por diferentes medios y por profesores/as de diversa preparación o aptitudes, sin que pueda asegurarse el éxito exclusivamente en base a una gran preparación técnica o a unas excelentes cualidades humanas. Citemos como ejemplo el caso de la profesora que domina la L2 y la utiliza, conoce la técnica y la combinación de actividades centradas en el contenido con las centradas en la forma etc., pero que no es capaz de crear un entorno afectivo favorable por la perplejidad en que sume a sus alumnos/as cortando su flujo comunicativo, interfiriendo en sus producciones y contradiciendo sus iniciativas. O el caso de otro profesor, técnicamente menos preparado pero buen motivador de la actividad grupal.

El profesor/a es, en definitiva, una variable multifactorial en interacción con otra variable multifactorial como es el grupo de aprendices, aspectos en los que también deberá centrarse la investigación sobre el proceso de aprendizaje de la lengua en los programas de inmersión.

\section{Referencias}

Allen, P.; SWAIN, M; Harley, B., y Cummins J. (1990) "Aspects of classroom treatement: toward a more comprehensive view of second language education" in Harley B., Allen P., Cummins J., Swain M. "The development of second language proficiency". Cambridge University Press.

ARNAU, J. (1993) "La experiencia de la inmersión" in "I Jornadas de Educación Plurilíngüe". Fundación Gaztelueta. Getxo.

ARTIGAL, J. M. (1993) "La importancia del uso en la adquisición de una nueva lengua" in "I Jornadas de Educación Plurilingüe". Fundación Gaztelueta. Getxo. p. 99-111.

BAKER, C. (1993) "Foundations of Blilingual Education and Bilingualism". Multilingual Matters. Clevedon.

CHAUdRON, C. (1986) "Teachers' Priorities in Correcting Learners' Errors in French Immersion Classes" in R. Day (Ed.) "Talking to Learn". Newbury House. Rowley. Mass.

Ews, R. (1985) “Understanding Second Language Acquisition". Oxford University Press.

Euss, R. (1992) "Second Language Acquisition and Language Pedagogy". Multilingual Matters. Clevedon.

HAMERS, J. F.,y Blanc, M. (1983) “Bilingualité et bilinguisme”. Ed. Pierre Mardaga. Bruxelles.

HARLEY, B. (1993) "Instructional Strategies and SLA in Early French Immersion". in Studies in Second Language Acquisition, Vol. 15, N² 2, Junio 1993, Cambridge University Press.

KraShen, S. (1982) "Principles and Practice in Second Language Acquisition". Pergamon. Oxford. 
Leeman Guthrie, E. (1987) "Six Cases in Clasroom Communication: A Study of Teacher Discourse in the Foreign Language Classroom". in Lantolf J.P., Labarca A. "Research in Second Language Learning: Focus on the Classroom". Ablex. Norwood.

PORTER, P. A. (1986) "How Leamers Talk to Each Other: Input and Interaction in Task-Centered Discussions" in R. Day (Ed.) "Talking to Learn". Newbury House. Rowley. Mass.

Rebuffor, J. (1993) "Le Point sur L'immersion au Canada". Centre Educatif et Culturel. Quebec.

SAFTY, A. (1991) "French immersion in Canada: theory and practice". in International Review of Education, 37 (4). p. 473-488.

SwaIN, M. (1988) "Manipulating and Complementing Content Teaching to maximize Second Language Learning". in TESL Canada Journal, vol. 6, No. 1, p. 68-83.

Thomas, W.; Coluter, V.,y ABBOTT, M. (1993) “Academic Achievement through Japanese, Spanish, or French: The First Two Years of Partial Immersion". in The Modern Language Journal, 77. 1993. p $.170-179$

Vח.A, I. (1993) “Aspectos didácticos de la educación plurilingüe” in "I Jornadas de Educación Plurilingüe". p. 159-172. Fundación Gaztelueta. Getxo.

WONG-FILLMORE, L. (1982) “ Instructional Language as Linguistic Input: Second-Language Learning in Classrooms" in Wilkinson L. C. (Ed.) "Communicating in the classroom". Academic Press. N.Y.

WONG-FILLMORE, L. (1985) "When does teacher talk work as input?" in Gass S. M., Madden C.G. "Input in second language acquisition". Newbury House. Rowley. Mass.

WONG-FILLMORE, L. (1991) "Second-language learning in children: a model of language learning in social context".in Bialystok E. (Ed.) "Language processing in bilingual children". Cambridge University Press. p. 49-69.

\section{Metodología y práctica en programas de inmersión Josu Sierra$$
\text { CL\&E, 1994, 22, pp. 85-95 }
$$

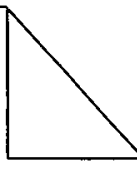

Resumen: Se citan, en primer lugar, los principios teóricos de los programas de inmersión, para, posteriormente, comentar las incidencias de la metodología aplicada según los estudios disponibles. Tras analizar los problemas derivados de la aplicación de la metodología de inmersión, se incide en las posibles soluciones y también se consideran otros factores al margen de los puramente metodológicos, tales como los de la estructura de la clase e, incluso, el profesor/a.

Datos sobre el autor: Josu Sierra es psicólogo y trabaja en el campo de la evaluación y la investigación sobre enseñanza bilingüe.

Dirección: Etxepare, 3, 48960 Galdakao (Bizkaia).

- C PERMISOS PARA CITAR O REPRODUCIR EN OTRAS FUENTES: Se pueden citar libremente hasta 500 palabras. Para reproducir una porción de texto mayor, figuras o ilustraciones, se deberá pedir permiso por escrito a la revista, especificando el uso al que se destina el texto. En todos los casos, se deberá citar el copyright de $C L \& E$. En el caso de artículos o textos que hayan sido a su vez reproducidos en $C L \& E$ los interesados deberán dirigirse tanto a los detentadores del copyright original como a $C L \& E$, en el caso de que se quiera hacer uso de la traducción. FO'TOCOPIAS: Para todo lo relacionado con el uso mediante fotocopia del material de esta revista, deberán dirigirse a: CEDRO, C/ José Marañón, 10, $3 .^{\circ}$ Izda. Tel. 5941575 . Fax 4453567 